\title{
Thermodinamic Interpretaion of the Morphology Individuality of Natural and Synthesized Apatite Single Crystals
}

\author{
Takaomi Suzuki, Haruka Takemae, Mika Yoshida
}

Department of Environmental Science and Technology, Faculty of Engineering, Shinshu University, Nagano, Japan.

Email: takaomi@shinshu-u.ac.jp

Received July $24^{\text {th }}, 2013$; revised August $23^{\text {rd }}, 2013$; accepted September $5^{\text {th }}, 2013$

Copyright (C) 2013 Takaomi Suzuki et al. This is an open access article distributed under the Creative Commons Attribution License, which permits unrestricted use, distribution, and reproduction in any medium, provided the original work is properly cited.

\begin{abstract}
Specific surface free energy (SSFE) of natural calcium fluorapatite from the same mother rock and synthesized barium chlorapatite from the same lot was determined using contact angle of water and formamide droplets, compared with grown length of crystal face $(h)$. The experimentally obtained SSFEs have different values even for the same index faces of the different crystals. The SSFEs also have wide distribution for each face of crystals. Observed SSFE is considered to be not only the SSFE of ideally flat terrace face, but also includes the contribution of strep free energy. Though the crystals we experimentally obtained were growth form, the relationship between SSFE and $h$ was almost proportional, which looks like satisfying Wulff's relationship qualitatively. The slope of SSFE versus $h$ line shows the driving force of crystal growth, and the line for larger crystal has less steep slope. The driving force of crystal growth for larger crystal is smaller, which also means that the chemical potential is larger for larger crystal. The individuality of crystals for the same lot can be explained by the difference of the chemical potential of each crystal.
\end{abstract}

Keywords: Crystal Morphology; Single Crystal Growth; Apatite Crystal

\section{Introduction}

Measurement of specific surface free energy (SSFE) of solid using contact angle of liquid is very popular and well accepted in the field of polymer science. Especially, automatic contact angle meter with software for the analysis of SSFE can be obtained commercially (for example, CA-V Series, Kyowa Interface Science). However, in the field of crystal science, the measurement of SSFE of crystals is believed to be difficult. In fact, experimental determination of SSFE of crystal is very few, even though the significance of SSFE for crystal growth is well discussed. Only a few trials to determine the surface energy of crystals were reported. For example, bonding energy of quartz was measured by introducing a crack into the crystal, and the energy of breaking was determined as the surface energy of the quartz crystal [1]. Determination of SSFE of polymer surface is not difficult because we can easily determine the contact angle with a good reproducibility. However, the contact angles of liquid on inorganic crystal face have very wide distribution, and we need to take a lot of photographs to de- termine the average of contact angle. We determined SSFE of barium chlorapatite single crystals from contact angle of liquids [2] for the first time. At that time we used more than 4000 photographs in order to determine the average contact angle of liquids. We also determined the SSFE of ruby crystals from the contact angle of liquid droplet and discussed the relationship between the grown length of the crystal face [3]. Although the ruby crystals were growth shape, the relationship between the SSFE and $h$ were almost proportional, which looks satisfying Wulff's relationship. However, Wulff's relationship should be satisfied for ideal equilibrium system, and the crystal face should be ideally flat. At this time, we extended our experimental technique for natural mineral crystals in order to study the relationship between SSFE and $h$ for the less ideal but more real system.

\section{Experimental Procedure}

Natural calcium fluorapatite crystals (Cerro del Mercado Cd. Durango, Mexico) were produced from a cluster of crystals with mother rock using a hammer and a chisel. 
The mother rock is fragile and the interface between the crystal and the mother rock can be easily separated. The advantage of this sample is that a clean and as-grown flat crystal face can be obtained. Ideal form of the fluorapatite crystal is hexagonal prism with pyramidal end faces. The indices of the prismatic faces is $\{10 \overline{1} 0\}$ and that of the pyramidal faces is $\{10 \overline{1} 1\}$.

Four clean crystals without serious damage were obtained from a mother rock and named crystal (A), (B), (C), and (D) (Figure 1). Because not all the faces of the crystal look clean and flat, the cleanest face in the $\{10 \overline{1} 1\}$ faces is determined as $(10 \overline{1} 1)$ face and used for the experiment. Also the adjacent $(10 \overline{1} 0)$ face of each crystal was used. Crystals of barium chlorapatite were synthesized using $\mathrm{NaCl}$ flux method [4]. Three well formed crystals were named crystal (a), (b), and (c) (Figure 2).

The crystals were sonicated with ethanol and thoroughly dried. Each single crystal was fixed on a glass plate using a small piece of clay for contact angle measurement. Water and formamide was dropped onto the crystal using a micropipette. The droplets with a volume of $\approx 0.1 \mathrm{~mm}^{3}$ were observed using a digital camera
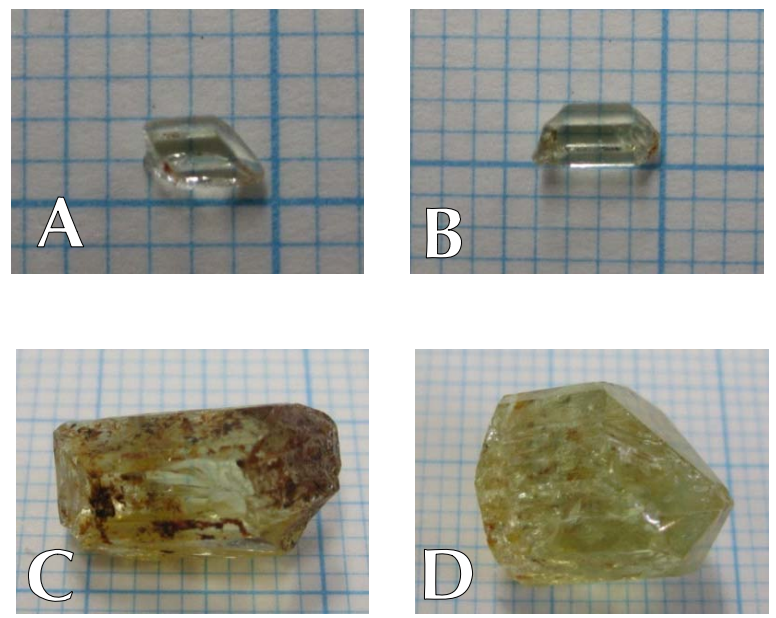

Figure 1. Photographs of natural fluorapatite crystals (A), (B), (C), and (D). The scale of the paper under the crystal is $1 \mathrm{~mm}$. The larger crystal is more opaque.
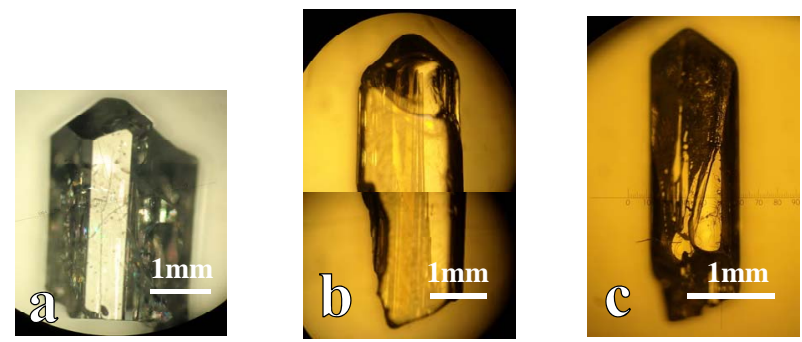

Figure 2. Photographs of synthesized chlorapatite crystals (a), (b), and (c). with a magnifying lens. The details of measurement of the contact angles of the droplets are described elsewhere [2].

\section{Results}

The values of SSFE, $\gamma_{\mathrm{s}}$, can be calculated from the obtained contact angle of the liquid, $\theta$, using the Fowkes approximation [5] and Wu's harmonic mean equations [6].

The polar and dispersed components of the liquid were $\gamma_{\mathrm{LV}}^{\mathrm{d}}=22.1 \mathrm{mN} / \mathrm{m}, \quad \gamma_{\mathrm{LV}}^{\mathrm{p}}=50.7 \mathrm{mN} / \mathrm{m}$ for water and $\gamma_{\mathrm{LV}}^{\mathrm{d}}=39.5 \mathrm{mN} / \mathrm{m}, \quad \gamma_{\mathrm{LV}}^{\mathrm{p}}=18.7 \mathrm{mN} / \mathrm{m}$ for formamide, which were taken from reported data [7].

The length of the normal line from the center of the crystal to each face, $h_{i}$, was obtained from the photograph and the $h_{i}$ was considered to be the grown length of the $i$ th face. The SSFE of natural calcium fluorapatite was compared with $h_{i}$ and shown in Figure 3. The line joining the points of $(10 \overline{1} 0)$ and $(10 \overline{1} 1)$ faces of crystal (A) in Figure 3 almost passes through the origin. On the other hand, the slope of the line is less steep for larger crystals. The relationship between calculated SSFE of synthesized barium chlorapatite (a), (b), and (c) are shown in Figure 4 with the plots of natural calcium fluorapatite (A)-(D). They are found between the plots of crystal (A) and (B).

\section{Theoretical Background}

Surface free energy of a crystal is a sum of the free energy of each face as,

$$
G_{\text {surf }}=\sum A_{i} \gamma_{i}
$$

where $\gamma_{i}$ is the SSFE and $A_{i}$ is the surface area of $i$ th

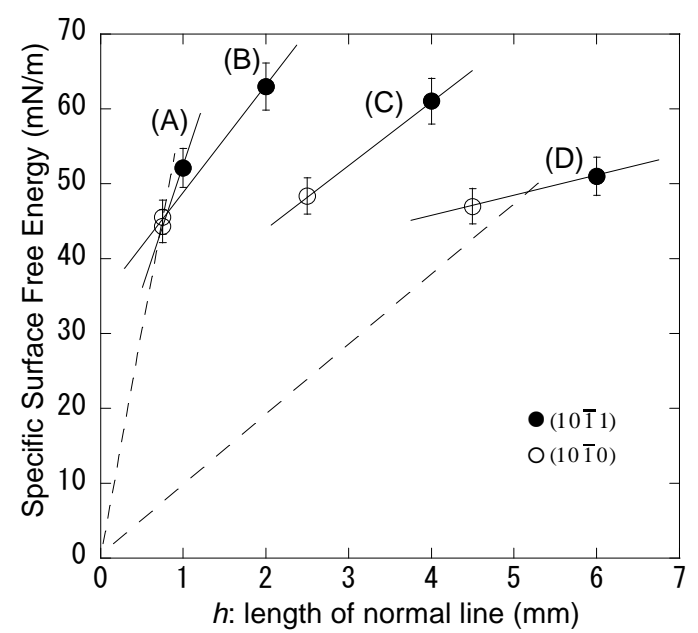

Figure 3. Relationship between the specific surface free energy and the length of the normal line to each face of natural fluorapatite crystals (A), (B), (C), and (D). 


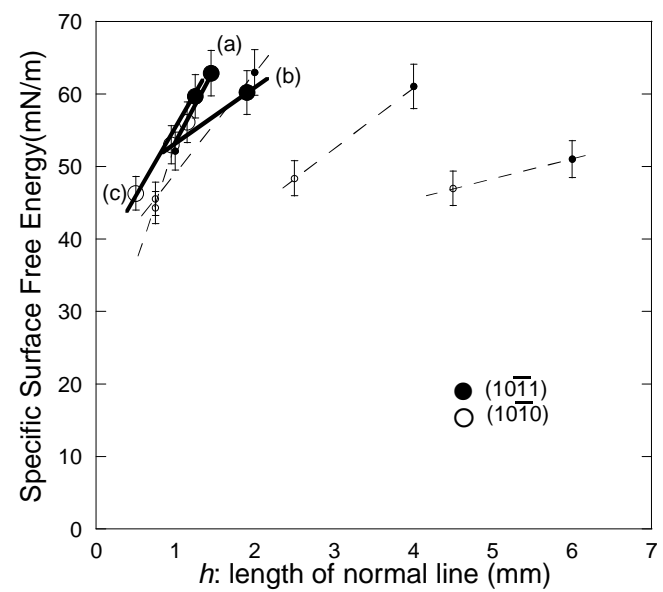

Figure 4. Relationship between the specific surface free energy and the length of the normal line to each face of chlorapatite crystals (a), (b), and (c). Plots of natural fluorapatite crystals are also shown by small marks.

face. The equilibrium shape of the crystal is determined by minimization of the surface free energy, $G_{\text {surf }}$, and well known Wulff's relationship [8] is obtained as,

$$
\gamma_{i} / h_{i}=\Delta \mu / 2 v_{s}=\text { constant }
$$

where $\Delta \mu$ is the driving force of crystal growth, and $v_{S}$ is atomic volume of the crystal. If the crystals were ideally equilibrium, the relationship between the SSFE, $\gamma_{i}$, and the grown length of the crystal, $h_{i}$, satisfies Equation (2). Therefore, all crystals in the same system should be the similar. Also, if the crystal face is ideally flat, like terrace, the SSFE of the same index face should be the same for different crystals. Therefore, the crystal habit must be the same for the equilibrium system.

\section{Discussion}

Though ideal equilibrium crystal shape is uniform, real crystals from the same lot have different shape and they are called growth shape. Our experimental result shows that the SSFE of the same index face for the different crystals are not the same, and the contact angles of liquid for one individual crystal face have wide distribution, which corresponds to the wide distribution of SSFE of a crystal face. Such wide distribution of observed SSFE can be caused by step free energy. The real crystals have not only ideal flat terrace face, but also steps are distributed on the surface of crystal face. The observed SSFE, $\gamma_{i}^{\text {obs }}$, can be described as,

$$
\gamma_{i}^{\text {obs }}=\gamma_{i}^{\text {terr }}+L \beta
$$

where $\gamma_{i}^{\text {terr }}$ is SSFE of ideally flat face, $L$ is step density, and $\beta$ is step free energy. In the real system, the surface free energy of crystal can be described as,

$$
G_{\text {surf }}^{\text {real }}=\sum A_{i} \gamma_{i}^{\text {obs }}
$$

and the minimization of $G_{\text {surf }}^{\text {real }}$ requires the relationship as ,

$$
\gamma_{i}^{\text {obs }} / h_{i}=\left(\gamma_{i}^{\text {terr }}+L \beta\right) / h_{i}=\text { constant }
$$

The distribution of the step density is considered to cause the wide distribution of the observed SSFE of the crystal. Also, if each crystal satisfies the thermodynamic equilibrium and stable state, and the individuality of each crystal can be explained by the difference of the step density distribution. The line binding the plot of $(10 \overline{1} 0)$ and (1011) of crystal (A) passes a point near origin. Crystal (A) is most close to equilibrium condition, because crystal (A) is the smallest, and it satisfied Equation (5). On the other hand, the lines binding the plot of $(10 \overline{1} 0)$ and $(10 \overline{1} 1)$ of larger crystals are apart from origin, because they are apart from equilibrium, and hardly satisfy Equation (5). However, the slope of lines drawn from origin to the center of points between $(10 \overline{10})$ and $(10 \overline{1} 1)$ which is shown by broken lines in Figure 3 roughly correspond to the constant of $\Delta \mu / 2 v_{S}$ of Equation (2). For example, the slope of line for crystal (D) is less steep than that of crystal (A), indicating the relationship between the driving force of crystal (A), $\Delta \mu_{\text {(A) }}$, and that of crystal (D), $\Delta \mu_{(\mathrm{D})}$, is described as,

$$
\Delta \mu_{(\mathrm{A})}>\Delta \mu_{(\mathrm{D})}
$$

Because both crystals are produced from the same mother rock, the chemical potential of liquid phase should be the same. The driving force of both crystal can be described as,

$$
\Delta \mu_{(\mathrm{A})}=\mu^{l i q}-\mu_{(\mathrm{A})}^{\text {sol }}
$$

and

$$
\Delta \mu_{(\mathrm{D})}=\mu^{\text {liq }}-\mu_{(\mathrm{D})}^{\text {sol }}
$$

where $\mu^{\text {liq }}, \mu_{(\mathrm{A})}^{\text {sol }}$, and $\mu_{(\mathrm{D})}^{\text {sol }}$ is the chemical potential of liquid phase, that of crystal (A) and (D), respectively. The relationship of Equation (6) requires the relationship as,

$$
\mu_{(\mathrm{A})}^{\text {sol }}<\mu_{(\mathrm{D})}^{\text {sol }}
$$

The chemical potential for larger crystal should be larger than that for smaller crystal. The larger chemical potential is considered to be resulted from the defects or contamination in the crystal. In fact, the photographs of crystals in Figure 1 show that the larger crystals look dirtier than the smaller crystals. This relationship is schematically described in Figure 5, where dots and lines in the picture indicate contamination and defects. Such contamination and defects make the crystal unsta- 


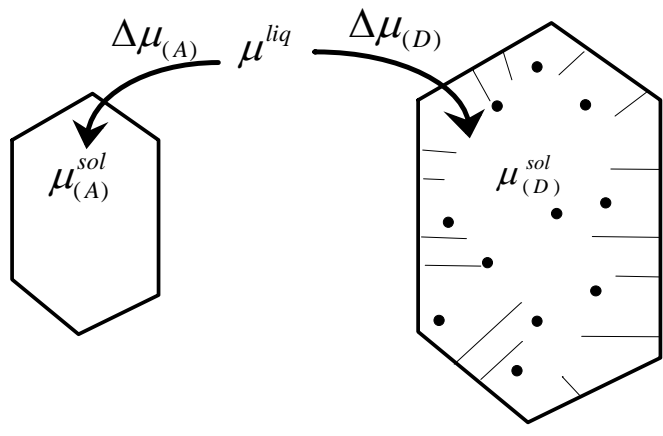

Figure 5. Schematic picture of relationship between driving force and the chemical potential of crystals. The dots and lines in crystal (D) indicate contamination and defects. Because of such contamination and defects, the crystal (D) has larger chemical potential than crystal (A).

ble, and the energy of the bulk crystal is higher. Therefore, the chemical potential of the crystal (D), $\mu_{(\mathrm{D})}^{\text {sol }}$, is larger than that of (A), $\mu_{(\mathrm{A})}^{\text {sol }}$, as Equation (9). The SSFE of synthesized barium chlorapatite (a)-(c) were also qualitatively satisfied relationship of Equation (5). The SSFE for $(10 \overline{1} 0)$ is larger than that for $(10 \overline{1} 1)$. The relationship between SSFE and $h$ is close to natural crystals (A) and (B).

\section{Conclusion}

Though the crystals from the same mother rock or the same crucible have a variety of shapes, their shapes are resulted from the thermodynamic equilibrium. The observed SSFE was almost proportional to the grown length of the face, and the individuality of the crystal shape is explained by the minimization of surface free energy including step free energy.

\section{REFERENCES}

[1] W. F. Brace and J. B. Walsh, American Mineralogist, Vol. 47, 1962, p. 1111.

[2] T. Suzuki, K. Nakayama and S. Oishi, Bulletin of the Chemical Society of Japan, Vol. 77, 2004, p. 109. http://dx.doi.org/10.1246/bcsj.77.109

[3] T. Suzuki and M. Oda, "Specific Surface Free Energy and the Morphology of Synthesized Ruby Single Crystals," Journal of Crystal Growth, Vol. 318, No. 1, 2011, pp. 76-78. http://dx.doi.org/10.1016/j.jcrysgro.2010.11.058

[4] S. Oishi, N. Michiba, N. Ishizawa, J. C. Rendon-Angeles and K. Yanagisawa, Bulletin of the Chemical Society of Japan, Vol. 72, 1999, p. 2097. http://dx.doi.org/10.1246/bcsj.72.2097

[5] F. M. Fowkes, “Attractive Forces at Interfaces,” Industrial \& Engineering Chemistry Research, Vol. 56, No. 12, 1954, pp. 40-52. http://dx.doi.org/10.1021/ie50660a008

[6] S. Wu, "Calculation of Interfacial Tension in Polymer Systems,” Journal of Polymer Science Part C, Vol. 34, No. 1, 1971, pp. 19-30. http://dx.doi.org/10.1002/polc.5070340105

[7] R. N. Shimizu and N. R. Demarquette, "Evaluation of Surface Energy of Solid Polymers Using Different Models,” Journal of Applied Polymer Science, Vol. 76, No. 12, 2000, pp. 1831-1845. http://dx.doi.org/10.1002/(SICI)1097-4628(20000620)76: 12<1831::AID-APP14>3.0.CO;2-Q

[8] G. Wulff, Zeitschrift für Kristallographie, Vol. 34, 1901, p. 449. 